\title{
Effect of ion beam on electromagnetic ion cyclotron instability in hot anisotropic plasma-particle aspect analysis
}

\author{
S. Patel, P. Varma, M. S. Tiwari, and N. Shukla \\ Department of Physics, Dr. H.S.Gour Central University, Sagar (M.P.) - 470003, India \\ Received: 24 January 2011 - Revised: 18 August 2011 - Accepted: 22 August 2011 - Published: 30 August 2011
}

\begin{abstract}
Using the general loss-cone distribution function electromagnetic ion cyclotron (EMIC) instability affected by up going ion beam has been studied by investigating the trajectories of charged particles. The plasma consisting of resonant and non-resonant particles has been considered. It is assumed that the resonant particles participate in energy exchange with the wave, whereas non-resonant particles support the oscillatory motion of the wave. The effect of ion beam velocity on the dispersion relation, growth rate, parallel and perpendicular resonant energy of the EMIC wave with general loss-cone distribution function in hot anisotropic plasma is described by particle aspect approach. The effect of beam anisotropy and beam density on electromagnetic ion cyclotron instabilities is investigated. Growth length is derived for EMIC waves in hot anisotropic plasma. It is found that the effect of the ion beam is to reduce the energy of transversely heated ions, whereas the thermal anisotropy of the background plasma acts as a source of free energy for the EMIC wave and enhances the growth rate. It is observed that ion beam velocity opposite to the wave propagation and its density reduces the growth rate and enhance the reduction in perpendicularly heated ions energy. The effect of ion beam anisotropy on EMIC wave is also discussed. These results are determined for auroral acceleration region. It is also found that the EMIC wave emissions occur by extracting energy of perpendicularly heated ions in the presence of an up flowing ion beam.
\end{abstract}

Keywords. Magnetospheric physics (Plasma waves and instabilities)

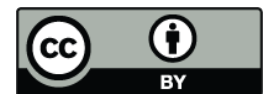

Correspondence to: P. Varma

(poornimavarma@yahoo.com)

\section{Introduction}

Electromagnetic ion cyclotron waves are a common and important feature of the Earth's magnetosphere. The source of free energy for wave excitation is provided by the temperature anisotropy of pitch angle distribution of ring current ions, which naturally develops during inward convection from the plasmasheet (Gamayunov et al., 2009). The EMIC waves have frequencies below the proton gyrofrequency, and they are excited mainly in the vicinity of the magnetic equator with a quasi-field-aligned wave normal angle (Kennel and Petschek, 1966). These waves were observed in the inner (Erlandson and Ukhorskiy, 2001) and outer (Anderson et al., 1992a, b) magnetosphere, at geostationary orbit (Mauk, 1982), at high latitudes (Erlandson et al., 1990) and at ionospheric altitudes (Braysy et al., 1998).

Usanova et al. (2008) have stated that Pc1 $(0.2-5 \mathrm{~Hz})$ pulsations are continuous geomagnetic field fluctuations believed to be generated by the electromagnetic ion cyclotron instability. The instability causes pitch angle diffusion, proton precipitation, and generation of ion cyclotron waves. Engebretson et al. (2007) observed that ion cyclotron wave growth rates could be significantly increases by addition of cool hydrogen (Sandanger et al., 2007).

The hot and cold plasma populations in the inner magnetosphere interact with each other. Energy transfer between the ring current and the plasmasphere cause decay of the hot population and heating in the cold plasma (Kozyra et al., 1987; Fok et al., 1993, 2005). Anisotropies in ring current and radiation belt particles excite plasma wave growth in the plasmasphere. These waves in turn, give rise to pitch-angle and energy diffusion of the energetic plasma (Jardanova et al., 2001; Khazanov et al., 2003; Summers et al., 1998). Energy is transferred from the hot ions to the plasmaspheric particles. The hot ions thus lose energy and when their energies are reduced to below the ring current energy range $(<1 \mathrm{keV})$, these ions are treated as lost from the ring current.

Collin et al. (1998) stated that the most intense visual auroral displays are the result of a series of complicated processes

Published by Copernicus Publications on behalf of the European Geosciences Union. 
that include acceleration of electrons downwards and ions upwards so called auroral acceleration region. Newell et al. (1998) have identified unexpected variations in the occurrence of intense auroral displays in the evening local time sector. These exciting new results, if independently confirmed, can provide new insights into some of the processes involved in the formation of auroral displays (Collin et al., 1998). The importance of ion cyclotron waves in auroral physics lies in their ability to heat ions, accelerate/modulate electrons, and perhaps to provide anomalous resistively, allowing for the creation of a parallel potential drop (Ahirwar et al., 2006b). Observations from Fast Auroral Snapshot (FAST) spacecraft at $\sim 4000 \mathrm{~km}$ altitude in ion beam region (Cattell et al., 1991; Ergun et al., 1998) show that these waves are electromagnetic. In past studies on EMIC instability, in auroral acceleration region have been performed by various authors (Gomberoff and Elgueta, 1991; Horne and Thorne, 1997; Ahirwar et al., 2006b, 2007). The up going ion beam are reported by the S3-3 satellite (Mozer et al., 1977) and by Polar satellite data (Mozer and Hull, 2001). Ions are often observed to have been accelerated transversely to the background magnetic field in the auroral region (Sharp et al., 1977; Lund et al., 2001). As they drift upward in the diverging field ions exchange some of their perpendicular energy via the mirror force to the energy of motion along the field. Such distributions, known as ion conics, have their characteristic shape in velocity space (Lund et al., 2001). The association of ion conics with EMIC waves has been given by various workers (Thorne and Horne, 1997; Lund et al., 1999a, b, c). Here we concentrate upon the excitation of EMIC waves by an up flowing ion beam in the converging field of the auroral acceleration region and the energy exchange of the ions along and perpendicular to the magnetic field considering only $\mathrm{H}^{+}$ions to examine behavior of EMIC waves.

Ion beam is commonly believed to be produced by acceleration through a field aligned potential drop which also accelerates electrons downwards producing "inverted V" electron distributions. Collin et al. (1986) showed that the ion beam energy is closely related to the magnitude of the potential drop estimated from the enhancement of the electron loss cones. In order to examine up flowing ions only near the auroral acceleration region, data were used from only near perigee which was at an altitude of about $0.8 R_{\mathrm{E}}$ over the southern polar region (Collin et al., 1998). Ahirwar et al. (2006) have stated that the ion beam considered does not follow the loss-cone distribution, it is background plasma of the auroral acceleration region which may permit the general loss-cone distribution due to converging magnetic field lines. The ion beam is supposed to follow the drifting general losscone distribution function (Gomberoff, 1992). The ion beam in the direction of the wave motion may damp these waves if the ion beam velocity is smaller than the phase velocity of the wave; however, the ion beam opposite to the wave motion may excite the waves, as reported in this paper. In our present paper, we have considered the electrodynamics of the auroral ionospheric region by an EMIC wave study. The theoretical analysis so developed may be extended to ring current and to the equatorial region of the space plasma.

In the past Ahirwar et al. (2007) have considered the effect of ion-beam velocities on EMIC waves with general loss-cone distribution function and have examine the effects of beam velocities and loss-cone distribution induces $J$. They have used the cold-plasma dispersion relation which may be inappropriate as plasma is thermalised and thermal anisotropies play a significant role. Ahirwar et al. (2007) have not distinguished between the beam plasma and the background core plasma adopting the same distribution function for both the plasmas which may be inadequate for the analysis. In the present paper we have considered the hot plasma dispersion relation and have used separate distribution function for core plasma and the ion beam. The effects of beam densities and beam anisotropies have been also considered and growth length is obtained. The results so obtained are discussed for the auroral acceleration region.

The method adopted here knows as particle aspect analysis has been widely used in the analysis of electrostatic and electromagnetic instability (Duan et al., 2005; Mishra and Tiwari, 2006; Patel et al., 2011). The main advantage of this approach is to consider the energy transfer between waves and particles along with the discussion of dispersion relation and growth/damping rate of the wave. The method may be suitable to deal with the auroral electrodynamics where particle acceleration is also important along with wave emissions.

\section{Basic trajectories}

The waves propagating in the direction of ambient magnetic field along the $\mathrm{z}$-axis are considered. The EMIC waves are assumed to start at $t=0$ when the resonant particles are not yet disturbed. Taking the particle trajectory in the presence of EMIC waves, the dispersion relation, the change in the charged particle energy and growth rate are then derived for different distribution indices in the presence of up flowing ion beams, in anisotropic plasma by the particle aspect analysis.

The basic mathematical treatment of EMIC waves using the particle aspect approach is given by Misra and Tiwari (1979). The left-handed circularly polarized waves, having an angular frequency $\omega$, are considered as:

$B_{\mathrm{x}}=B \cos (k z-\omega t)$
$B_{\mathrm{y}}=B \sin (k z-\omega t)$

When the system is co-moving with the waves, the electric field vanishes. Thus the wave magnetic field has the form

$\boldsymbol{B}=B_{\mathrm{x}} \cos (k z) \hat{x}+B_{\mathrm{y}} \sin (k z) \hat{y}$

Where the following transformation has been performed:

$z^{\text {wave }}=z^{\text {lab }}-(\omega / k) t$

$v^{\text {wave }}=v^{\text {lab }}-(\omega / k)$ 
Since $c k / \omega \gg 1$, the magnetic field amplitude may be regarded in both system as identical. Using the equation of ion motion in the presence of the wave, Misra and Tiwari (1979), have derived the ion perturbed velocities as

$$
\begin{aligned}
\delta V_{\perp \alpha}= & \frac{h \Omega_{\alpha}\left(V_{\|, \alpha}-\omega / k\right)}{\left.k V_{\alpha}-\left(\omega-\Omega_{\alpha}\right)\right]}[\cos (k z-\omega t-\Psi) \\
& -\varepsilon \cos \left(k z-\omega t-\Psi-\left(k V_{\|, \alpha}-\left(\omega-\Omega_{\alpha}\right) t\right)\right] \\
\delta V_{\|, \alpha}= & \frac{-h \Omega_{\alpha} V_{\perp, \alpha}}{\left[k V_{\|, \alpha}-\left(\omega-\Omega_{\alpha}\right)\right]}[\cos (k z-\omega t-\Psi) \\
& -\varepsilon \cos \left(k z-\omega t-\Psi-\left(k V_{\|, \alpha}-\left(\omega-\Omega_{\alpha}\right) t\right)\right]
\end{aligned}
$$

Where $\varepsilon=0$ for non resonant and $\varepsilon=1$ for resonant particles, $V_{-\alpha}$ and $V_{\| \alpha}$ are the initial value of the velocities at $t=0, \alpha=i, e$ stands for ions and electron core plasma, $\boldsymbol{h}=\frac{\boldsymbol{B}}{B_{0}}$ is the ratio of electromagnetic to static magnetic field amplitude, $\Omega_{\alpha}=\frac{q B_{0}}{m_{\alpha} c}$ is the cyclotron frequency and $\psi$ is the initial phase in perpendicular velocity.

\section{Density perturbations}

The perturbed density $n_{1}$ is obtained by the method outlined in reference (Ahirwar et al., 2006a, 2007)

$n_{c, \alpha}=\frac{h \Omega_{\alpha} V_{\perp, \alpha} k N(V)}{\left[k V_{\|, \alpha}-\left(\omega-\Omega_{\alpha}\right)\right]^{2}}\left[\cos \chi-\varepsilon \cos \chi_{0}+\varepsilon t A \sin (\chi-A t)\right]$

Where, $\chi=k z-\omega t-\psi, A=k V_{\|, \alpha}-\left(\omega-\Omega_{\alpha}\right)$.

\section{Distribution function}

We choose the general loss-cone distribution function for electron and ion core of background plasma

$N_{c}(V)=\frac{n_{c, \alpha} V_{\perp, \alpha}^{2 J}}{\pi^{3 / 2} V_{T \perp, \alpha}^{2(J+1)} V_{T \|, \alpha} J !} \exp \left(-\frac{V_{\perp}^{2}}{V_{T \perp, \alpha}^{2}}-\frac{V_{\|}^{2}}{V_{T \|, \alpha}^{2}}\right)(6)$

Where, $V_{T \|, \alpha}^{2}=\frac{2 T_{\|, \alpha}}{m}, \quad V_{T \perp, \alpha}^{2}=(J+1)^{-1} \frac{2 T_{\perp, \alpha}}{m}, \quad \alpha=i, e$ stands for ion and electron core plasma.

The drifting general loss-cone distribution function for ion beam (Gomberoff, 1992) is defined as,

$$
N_{\mathrm{b}}(V)=\frac{n_{\mathrm{b}} V_{\perp, \mathrm{b}}^{2 J}}{\pi^{3 / 2} V_{T \perp \mathrm{b}}^{2(J+1)} V_{T \| \mathrm{b}} J !} \exp \left(-\frac{V_{\perp}^{2}}{V_{T \perp \mathrm{b}}^{2}}-\frac{\left(V_{\|}-V_{\mathrm{D}}\right)^{2}}{V_{T \| \mathrm{b}}^{2}}\right)
$$

Where, $V_{\mathrm{D}}$ is the velocity of drifting beam related to the background core plasma. $V_{T \perp \mathrm{b}}$ and $V_{T|| \mathrm{b}}$ represent perpendicular and parallel thermal velocities of the ion beam.

\section{Dispersion relation}

The dispersion equation for hot plasma for the electromagnetic ion cyclotron wave (Cornwall and Schulz, 1971; Gomberoff, 1992; Patel et al., 2011) in auroral acceleration region are defined in the magnetic field as

$\frac{c^{2} k^{2}}{\omega^{2}}=\frac{\omega_{\mathrm{pi}}^{2}}{\Omega_{\mathrm{i}}\left(\Omega_{\mathrm{i}}-\omega\right)}+\frac{\omega_{\mathrm{pe}}^{2}}{\omega^{2}} A_{\mathrm{e}}+\frac{\omega_{\mathrm{pi}}^{2}}{\omega^{2}} \frac{n_{\mathrm{b} /} n_{c}\left(\omega-k V_{\mathrm{D}}\right)^{2}}{\Omega_{\mathrm{i}}\left(\Omega_{\mathrm{b}}-\omega+k V_{\mathrm{D}}\right)}$

Where, $\omega_{\mathrm{pe}}^{2}=\frac{4 \pi n_{\mathrm{e}} e^{2}}{M_{\mathrm{e}}}$ defines the plasma frequency for the electrons, $\omega_{\mathrm{pi}}^{2}=\frac{4 \pi n_{\mathrm{i}} e^{2}}{M_{\mathrm{i}}}$ defines the plasma frequency for the ions, $A_{\mathrm{e}}=(J+1) \frac{V_{T \perp \mathrm{e}}^{2}}{V_{T \| \mathrm{e}}^{2}}-1$ is the thermal anisotropies of electrons (Gomberoff and Cuperman, 1981).

\section{Energy balance}

The energy of the electromagnetic wave $U$ for the plasma is defined by the expression as, (Misra and Tiwari, 1979; Patel et al., 2011)

$U=\left(\frac{1}{16 \pi}\right)\left[\frac{d}{d \omega}\left(\omega \varepsilon_{\mathrm{i} k}\right) E_{\mathrm{i}}^{*} E_{k}+|B|^{2}\right]$

Where $\varepsilon_{i k}$ is the dielectric tensor. After the calculation the electromagnetic wave energy per unit wavelength is given by:

$$
\begin{aligned}
U= & \frac{\lambda B^{2}}{8 \pi} \omega_{\mathrm{pi}}^{2}\left[\frac{1}{\left(\Omega_{\mathrm{i}}-\omega\right)^{2}}+\frac{\left(n_{\mathrm{b}} / n_{c}\right)\left(\omega-k V_{\mathrm{D}}\right)}{\Omega_{\mathrm{b}}}\right. \\
& \left.\left\{\frac{\omega \Omega_{\mathrm{b}}-k V_{\mathrm{D}} \omega+k V_{\mathrm{D}} \Omega_{\mathrm{b}}+k^{2} V_{\mathrm{D}}^{2}}{\omega^{2}\left(\Omega_{\mathrm{b}}-\omega+k V_{\mathrm{D}}\right)^{2}}\right\}-\frac{\omega_{\mathrm{pe}}^{2}}{\omega_{\mathrm{pi}}^{2}} \frac{A_{\mathrm{e}}}{\omega^{2}}\right]+\frac{\lambda B^{2}}{8 \pi}
\end{aligned}
$$

The total wave energy per unit wave length $\lambda$ is given by,

$W_{W}=U+W_{\alpha}$

Where,

$$
\begin{aligned}
W_{\alpha}= & \int_{0}^{\lambda} d z \int_{0}^{2 \pi} d \psi \int_{0}^{\infty} V_{\perp 0} d V_{\perp 0} \int_{-\infty}^{\infty} d V_{\| 0} \frac{m}{2} \\
& {\left[\left(N+n_{1}\right)(V+\delta V)^{2}-N V^{2}\right] \alpha }
\end{aligned}
$$

We find the parallel $W_{\alpha \| c}$ and perpendicular $W_{\alpha \perp c}$ nonresonant energy with $\varepsilon=0$, by Eqs. (4), (5), (6) and (9) for background plasma, (Ahirwar et al., 2006a, 2007; Patel et al., 2011)

$W_{\| c, \alpha}=-\frac{\lambda B^{2}}{8 \pi} \frac{C_{J}}{V_{T \| \alpha}^{2}} \frac{\omega_{p \alpha}^{2}}{c^{2} k^{2}}\left[\frac{1}{2} Z_{1}\left(\xi_{\alpha}\right)+\frac{\omega-\Omega_{\alpha}}{k V_{T \| \alpha}} Z_{2}\left(\xi_{\alpha}\right)\right]$

Where, $Z\left(\xi_{\alpha}\right)=\frac{1}{\sqrt{\pi}} \int_{-\infty}^{\infty} \frac{\exp \left(-x^{2}\right)}{\left(x-\xi_{\alpha}\right)} d x$

$Z_{1}\left(\xi_{\alpha}\right)=\frac{1}{\sqrt{\pi}} \int_{-\infty}^{\infty} \frac{\exp \left(-x^{2}\right)}{\left(x-\xi_{\alpha}\right)^{2}} d x$ 
$Z_{2}\left(\xi_{\alpha}\right)=\frac{1}{\sqrt{\pi}} \int_{-\infty}^{\infty} \frac{\exp \left(-x^{2}\right)}{\left(x-\xi_{\alpha}\right)^{3}} d x, \quad \xi_{\alpha}=\frac{\omega-\Omega_{\alpha}}{k V_{T \| \alpha}}$

Similarly the perpendicular non resonant energy is given by the expression

$$
\begin{aligned}
W_{\perp c, \alpha}= & \frac{\lambda}{2} \frac{B^{2}}{8 \pi} \frac{\omega_{p \alpha}^{2}}{c^{2} k^{2}}\left[D_{J}\left(1-\frac{2 \Omega_{\alpha}}{k V_{T \| \alpha}} Z\left(\xi_{\alpha}\right)+\frac{\Omega_{\alpha}^{2}}{k V_{T \| \alpha}^{2}} Z_{1}\left(\xi_{\alpha}\right)\right)\right. \\
& \left.+\frac{2 C_{J}}{V_{T \| \alpha}^{2}}\left(Z_{1}\left(\xi_{\alpha}\right)-\frac{\Omega_{\alpha}}{k V_{T \| \alpha}} Z_{2}\left(\xi_{\alpha}\right)\right)\right]
\end{aligned}
$$

And parallel, perpendicular non resonant energies of beam is,

$$
\begin{aligned}
W_{\| b}= & -\frac{\lambda B^{2}}{8 \pi} \frac{C_{J}}{V_{T \| \mathrm{b}}^{2}} \frac{n_{\mathrm{b}}}{n_{c}} \frac{\omega_{\mathrm{pi}}^{2}}{c^{2} k^{2}} \\
& {\left[\frac{1}{2} Z_{1}\left(\zeta_{\mathrm{b}}-\frac{V_{\mathrm{D}}}{V_{T \| \mathrm{b}}}\right)+\frac{\omega-\Omega_{\mathrm{b}}}{k V_{T \| \mathrm{b}}} Z_{2}\left(\zeta_{\mathrm{b}}-\frac{V_{\mathrm{D}}}{V_{T \| \mathrm{b}}}\right)\right] } \\
W_{\perp \mathrm{b}}= & \frac{\lambda}{2} \frac{B^{2}}{8 \pi} \frac{\omega_{\mathrm{pi}}^{2}}{c^{2} k^{2}} \frac{n_{\mathrm{b}}}{n_{c}}\left[D _ { J } \left(1-\frac{2 \Omega_{\mathrm{b}}}{k V_{T \| \mathrm{b}}} Z\left(\zeta_{\mathrm{b}}-\frac{V_{\mathrm{D}}}{V_{T \| \mathrm{b}}}\right)\right.\right. \\
& \left.+\frac{\Omega_{\mathrm{b}}^{2}}{k V_{T \| \mathrm{b}}^{2}} Z_{1}\left(\zeta_{\mathrm{b}}-\frac{V_{\mathrm{D}}}{V_{T \| \mathrm{b}}}\right)\right)+\frac{2 C_{J}}{V_{T \| \mathrm{b}}^{2}} \\
& \left.\left(Z_{1}\left(\zeta_{\mathrm{b}}-\frac{V_{\mathrm{D}}}{V_{T \| \mathrm{b}}}\right)-\frac{\Omega_{\mathrm{b}}}{k V_{T \| \mathrm{b}}} Z_{2}\left(\zeta_{\mathrm{b}}-\frac{V_{\mathrm{D}}}{V_{T \| \mathrm{b}}}\right)\right)\right]
\end{aligned}
$$

Where, $Z\left(\zeta_{\mathrm{b}}-\frac{V_{\mathrm{D}}}{V_{T \| \mathrm{b}}}\right)=\frac{1}{\sqrt{\pi}} \int_{-\infty}^{\infty} \frac{\exp \left(-x^{2}\right)}{\left(x-\zeta_{\mathrm{b}}\right)} d x$

$Z_{1}\left(\zeta_{\mathrm{b}}-\frac{V_{\mathrm{D}}}{V_{T \| \mathrm{b}}}\right)=\frac{1}{\sqrt{\pi}} \int_{-\infty}^{\infty} \frac{\exp \left(-x^{2}\right)}{\left(x-\zeta_{\mathrm{b}}\right)^{2}} d x$

$Z_{2}\left(\zeta_{\mathrm{b}}-\frac{V_{\mathrm{D}}}{V_{T \| \mathrm{b}}}\right)=\frac{1}{\sqrt{\pi}} \int_{-\infty}^{\infty} \frac{\exp \left(-x^{2}\right)}{\left(x-\zeta_{\mathrm{b}}\right)^{3}} d x$, where $\zeta_{\mathrm{b}}=\frac{\omega-k V_{\mathrm{D}}-\Omega_{i}}{k V_{T \| \mathrm{b}}}$

$C_{J, \alpha}=\frac{2 \pi}{V_{T \perp}^{2(J+1)} J !} \int_{0}^{\infty} d V_{\perp}^{2} V_{\perp}^{2(J+1)} \exp \left(-\frac{V_{\perp}^{2}}{V_{T \perp \alpha}^{2}}\right)$

$D_{J, \alpha}=\frac{2 \pi}{V_{T \perp}^{2(J+1)} J !} \int_{0}^{\infty} d V_{\perp}^{2} V_{\perp}^{2 J} \exp \left(-\frac{V_{\perp}^{2}}{V_{T \perp \alpha}^{2}}\right)$

The total non resonant energy of core plasma and beam in parallel and perpendicular direction is

$W_{\|}=W_{\| c, \alpha}+W_{\| \mathrm{b}}$

$W_{\perp}=W_{\perp c, \alpha}+W_{\perp \mathrm{b}}$
The perpendicular and the parallel resonant energy of the core plasma, with $\varepsilon=1$ is,

$$
\begin{aligned}
W_{r \| c, \alpha}= & \frac{\sqrt{\pi}}{2} t \frac{B^{2} \omega_{p \alpha}^{2}}{c^{2} k^{2}} \frac{\Omega_{\alpha}^{2}}{k^{2} V_{T \| \alpha}}(J+1) \frac{V_{T \perp \alpha}^{2}}{V_{T \| \alpha}^{2}}\left(\frac{\omega-\Omega_{\alpha}}{\Omega_{\alpha}}\right)^{2} \\
& \exp \left(-\left(\frac{\omega-\Omega_{\alpha}}{k V_{T \| \alpha}}\right)^{2}\right) \\
W_{r \perp c, \alpha}= & \frac{\sqrt{\pi}}{2} t \frac{B^{2} \omega_{p \alpha}^{2}}{c^{2} k^{2}} \frac{\Omega_{\alpha}^{2}}{k^{2} V_{T \| \alpha}} \\
& {\left[(J+1) \frac{V_{T \perp \alpha}^{2}}{V_{T \| \alpha}^{2}}\left(\frac{\omega-\Omega_{\alpha}}{\Omega_{\alpha}}\right)+1\right] } \\
& \exp \left(-\left(\frac{\omega-\Omega_{\alpha}}{k V_{T \| \alpha}}\right)^{2}\right)
\end{aligned}
$$

For ion beam resonant parallel and perpendicular energy is defined as,

$$
\begin{aligned}
W_{r \| b}= & \frac{\sqrt{\pi}}{2} t \frac{B^{2} \omega_{\mathrm{pi}}^{2}}{c^{2} k^{2}} \frac{n_{\mathrm{b}}}{n_{c}} \frac{\Omega_{\mathrm{b}}^{2}}{k^{2} V_{T \| \mathrm{b}}} \\
& (J+1) \frac{V_{T \perp \mathrm{b}}^{2}}{V_{T \| \mathrm{b}}^{2}}\left(\frac{\omega-\mathrm{k} V_{\mathrm{D}}-\Omega_{\mathrm{b}}}{\Omega_{\mathrm{b}}}\right)^{2} \\
& \exp \left(-\left(\frac{\omega-k V_{\mathrm{D}}-\Omega_{\mathrm{b}}}{k V_{T \| \mathrm{b}}}\right)^{2}\right) \\
W_{r \perp \mathrm{b}}= & \frac{\sqrt{\pi}}{2} t \frac{B^{2} \omega_{\mathrm{pi}}^{2}}{c^{2} k^{2}} \frac{n_{\mathrm{b}}}{n_{c}} \frac{\Omega_{\mathrm{b}}^{2}}{k^{2} V_{T \| \mathrm{b}}} \\
& {\left[(J+1) \frac{V_{T \perp \mathrm{b}}^{2}}{V_{T \| \mathrm{b}}^{2}}\left(\frac{\omega-k V_{\mathrm{D}}-\Omega_{\mathrm{b}}}{\Omega_{\mathrm{b}}}\right)+1\right] } \\
& \exp \left(-\left(\frac{\omega-k V_{\mathrm{D}}-\Omega_{\mathrm{b}}}{k V_{T \| \mathrm{b}}}\right)^{2}\right)
\end{aligned}
$$

Total resonant energy is given as

$$
\begin{aligned}
& W_{r \|}=W_{r \| c, \alpha}+W_{r \| \mathrm{b}} \\
& W_{r \perp}=W_{r \perp c, \alpha}+W_{r \perp \mathrm{b}}
\end{aligned}
$$

The expressions for resonant energies Eqs. (12)-(17) depend upon $t$. In numerical calculations since the change in energy was evaluated per unit wave length the $t$ was replaced by $2 \pi / \omega$, hence the growth rate was evaluated.

\section{Growth rate}

From the energy conservation law, we can easily obtain an expression for the growth/damping rate $\gamma$ of the wave (Patel et al., 2011),

$\frac{d}{d t}\left[W_{r}+W_{W}\right]=0$ 
Thus, $\gamma=-\frac{d W_{r} / d t}{2 W_{W}}$.

Where, $W_{r}=W r_{-}+W r_{\|}, W_{W}=U+W_{i}$ and $W_{i}=W_{i-}+$ $W_{i \|}$.

Hence the growth rate is obtained as

$\frac{\gamma}{\Omega}=\frac{A+\left(n_{\mathrm{b}} / n_{c}\right) B}{c^{2} k^{2} C-\frac{c^{2} k^{2}}{\omega^{2}} \frac{\omega_{\mathrm{pe}}^{2}}{\omega_{\mathrm{pi}}^{2}} A_{\mathrm{e}}+\frac{c^{2} k^{2}}{\omega_{\mathrm{pi}}^{2}}+\frac{1}{2}\left(\frac{2 \Omega_{\mathrm{i}}-\omega}{\Omega_{\mathrm{i}}-\omega}\right)^{2}+\frac{n_{\mathrm{b}} / n_{c}}{2}\left(\frac{2 \Omega_{\mathrm{b}}-\omega^{\prime}}{\Omega_{\mathrm{b}}-\omega^{\prime}}\right)^{2}}$

$A=\frac{\Omega_{\mathrm{i}}}{k V_{T \| \mathrm{i}}}\left[\frac{\Omega_{\mathrm{i}}-\omega}{\Omega_{\mathrm{i}}}(J+1) \frac{V_{T \perp \mathrm{i}}^{2}}{V_{T \| \mathrm{i}}^{2}}-1\right] \exp \left(-\frac{\left(\omega-\Omega_{\mathrm{i}}\right)^{2}}{k^{2} V_{T \| \mathrm{i}}^{2}}\right)$

$B=\frac{\Omega_{\mathrm{b}}}{k V_{T \| \mathrm{b}}}\left[\frac{\Omega_{\mathrm{b}}-\omega^{\prime}}{\Omega_{\mathrm{b}}}(J+1) \frac{V_{T \perp \mathrm{b}}^{2}}{V_{T \| \mathrm{b}}^{2}}-1\right] \exp \left(-\frac{\left(\omega^{\prime}-\Omega_{\mathrm{b}}\right)^{2}}{k^{2} V_{T \| \mathrm{b}}^{2}}\right)$

$C=\left[\frac{1}{\left(\Omega_{\mathrm{i}}-\omega\right)^{2}}+\frac{\left(n_{\mathrm{b}} / n_{c}\right)\left(\omega^{\prime}\right)}{\Omega_{\mathrm{b}}}\left\{\frac{\omega \Omega_{\mathrm{b}}-k V_{\mathrm{D}} \omega+k V_{\mathrm{D}} \Omega_{\mathrm{b}}+k^{2} V_{\mathrm{D}}^{2}}{\omega^{2}\left(\Omega_{\mathrm{b}}-\omega+k V_{\mathrm{D}}\right)^{2}}\right\}\right]$

Where, $\omega^{\prime}=\omega-k V_{\mathrm{D}},(J+1) \frac{V_{T \perp \mathrm{i}}^{2}}{V_{T \| \mathrm{i}}^{2}}-1$ is the effective thermal anisotropy of core and $(J+1) \frac{V_{T \perp \mathrm{b}}^{2}}{V_{T \| \mathrm{b}}^{2}}-1$ is the effective thermal anisotropy of beam.

Here it is noticed that the ion beam $\left(V_{\mathrm{D}}\right)$ has affected the growth rate and change in energy for the electromagnetic ion cyclotron waves propagating parallel to the magnetic field with a general loss-cone distribution function for core plasma.

\section{Growth length}

The growth length of the electromagnetic ion cyclotron wave is derived from (Tiwari et al., 2008)

$L_{\mathrm{g}}=\frac{V_{\mathrm{g}}}{\gamma}$

Where, $\gamma$ is growth rate, $V_{\mathrm{g}}$ is group velocity of the wave.

$$
\begin{aligned}
& V_{\mathrm{g}}=\frac{d \omega}{d k} \\
& \frac{d \omega}{d k}=\frac{M}{N}
\end{aligned}
$$

Where

$$
\begin{aligned}
M= & \omega_{\mathrm{pi}}^{2}\left(\omega^{2} V_{\mathrm{D}}+2 \eta k V_{\mathrm{D}}^{2} \Omega_{\mathrm{i}}-2 \eta k V_{\mathrm{D}}^{2} \omega-2 \eta \omega V_{\mathrm{D}} \Omega_{\mathrm{i}}+2 \eta \omega^{2} V_{\mathrm{D}}\right) \\
& +\omega_{p e}^{2}\left(A_{e} \Omega_{i}^{2} V_{D}+A_{e} \Omega_{i}^{\omega} V_{\mathrm{D}}\right)+ \\
& \left(3 c^{2} k^{2} \Omega_{\mathrm{i}} V_{\mathrm{D}} \omega-3 c^{2} k^{2} \Omega_{\mathrm{i}}^{2} V_{\mathrm{D}}-2 c^{2} k^{2} \Omega_{\mathrm{i}}^{3}\right. \\
& \left.-4 c^{2} k \Omega_{\mathrm{i}}^{2} \omega-2 c^{2} k^{2} \Omega_{\mathrm{i}} \omega^{2}\right)
\end{aligned}
$$

$$
\begin{aligned}
N= & \omega_{\mathrm{pi}}^{2}\left(-2 \omega \Omega_{\mathrm{i}}+3 \omega^{2}-2 \omega \Omega_{i} k V_{\mathrm{D}}-2 \eta \omega \Omega_{\mathrm{i}}+3 \eta \omega^{2}\right. \\
& \left.+\eta k^{2} V_{\mathrm{D}}^{2}+2 \eta k V_{\mathrm{D}} \Omega_{\mathrm{i}}-4 \eta \omega k V_{\mathrm{D}}\right) \\
& +\omega_{\mathrm{pe}}^{2}\left(2 A_{\mathrm{e}} \Omega_{\mathrm{i}}^{2}-2 A_{\mathrm{e}} \Omega_{\mathrm{i}} \omega+A_{\mathrm{e}} \Omega_{\mathrm{i}} k V_{\mathrm{D}}\right) \\
& +\left(-2 c^{2} k^{2} \Omega_{\mathrm{i}}^{2}+2 c^{2} k^{2} \Omega_{\mathrm{i}} \omega-c^{2} k^{3} \Omega_{\mathrm{i}} V_{\mathrm{D}}\right)
\end{aligned}
$$

From Eq. (18)

$\gamma=\frac{P}{Q}$

Where

$$
\begin{aligned}
P= & \Omega_{\mathrm{i}}\left(A+\left(n_{\mathrm{b}} / n_{c}\right) B\right) \\
Q= & c^{2} k^{2} C-\frac{c^{2} k^{2}}{\omega^{2}} \frac{\omega_{\mathrm{pe}}^{2}}{\omega_{\mathrm{pi}}^{2}} A_{\mathrm{e}}+\frac{c^{2} k^{2}}{\omega_{\mathrm{pi}}^{2}}+\frac{1}{2}\left(\frac{2 \Omega_{\mathrm{i}}-\omega}{\Omega_{\mathrm{i}}-\omega}\right)^{2} \\
& +\frac{n_{\mathrm{b}} / n_{c}}{2}\left(\frac{2 \Omega_{\mathrm{b}}-\omega^{\prime}}{\Omega_{\mathrm{b}}-\omega^{\prime}}\right)^{2}
\end{aligned}
$$

Thus growth length is obtained from Eqs. (19), (20) and (21) as

$$
L_{\mathrm{g}}=\frac{M}{N} \times \frac{Q}{P}
$$

\section{Result and discussion}

In the numerical calculation of the growth rate, parallel and perpendicular resonant energies, we have used the following parameters for the auroral acceleration region plasma (Mishra and Tiwari, 2006; Ahirwar et al., 2006b; Gomberoff and Cuperman, 1981; Daughton and Gary, 1998)

$$
\begin{array}{ll}
B_{0}=4300 \mathrm{nT} & \Omega_{\mathrm{i}} \approx \Omega_{\mathrm{b}}=412 \mathrm{~s}^{-1} \\
a_{\mathrm{e}}=\frac{V_{T \perp \mathrm{e}}^{2}}{V_{T \| \mathrm{e}}^{2}}=0.1-2 & \\
T_{\mathrm{e}}=25-50 \mathrm{eV}, & V_{T \| \mathrm{i}}=6.41 \times 10^{8} \mathrm{~cm} \mathrm{~s}^{-1} \\
a_{\mathrm{i}}=\frac{V_{T \perp \mathrm{i}}^{2}}{V_{T \| \mathrm{i}}^{2}}=10-50 & \\
\omega_{\mathrm{pe}}^{2}=3.18 \times 10^{8} \mathrm{~s}^{-2} & \omega_{\mathrm{pi}}^{2}=1.732 \times 10^{6} \mathrm{~s}^{-2} \\
\eta=\frac{n_{\mathrm{b}}}{n_{c}}=\frac{3}{7} & a_{\mathrm{b}}=\frac{V_{T \perp \mathrm{b}}^{2}}{V_{T \| \mathrm{b}}^{2}}=1
\end{array}
$$

Equations (16)-(18), (22) have been evaluated numerically using Matlab Software to solve the growth rate and wave energies exchange between wave and particles.

The effect of the ion beam velocity $\left(V_{\mathrm{D}}\right)$, thermal electron anisotropy define by $A_{\mathrm{e}}=(J+1) \frac{V_{T \perp \mathrm{e}}^{2}}{V_{T \| \mathrm{e}}^{2}}-1$ and thermal ion anisotropy define by $A_{\mathrm{i}}=(J+1) \frac{V_{T \perp i}^{2}}{V_{T|| \mathrm{i}}^{2}}-1$ with different distribution indices $J$ on the waves growth rate and resonant energies is predicted. We have shown that EMIC wave particle interaction in the auroral acceleration region by graphical 

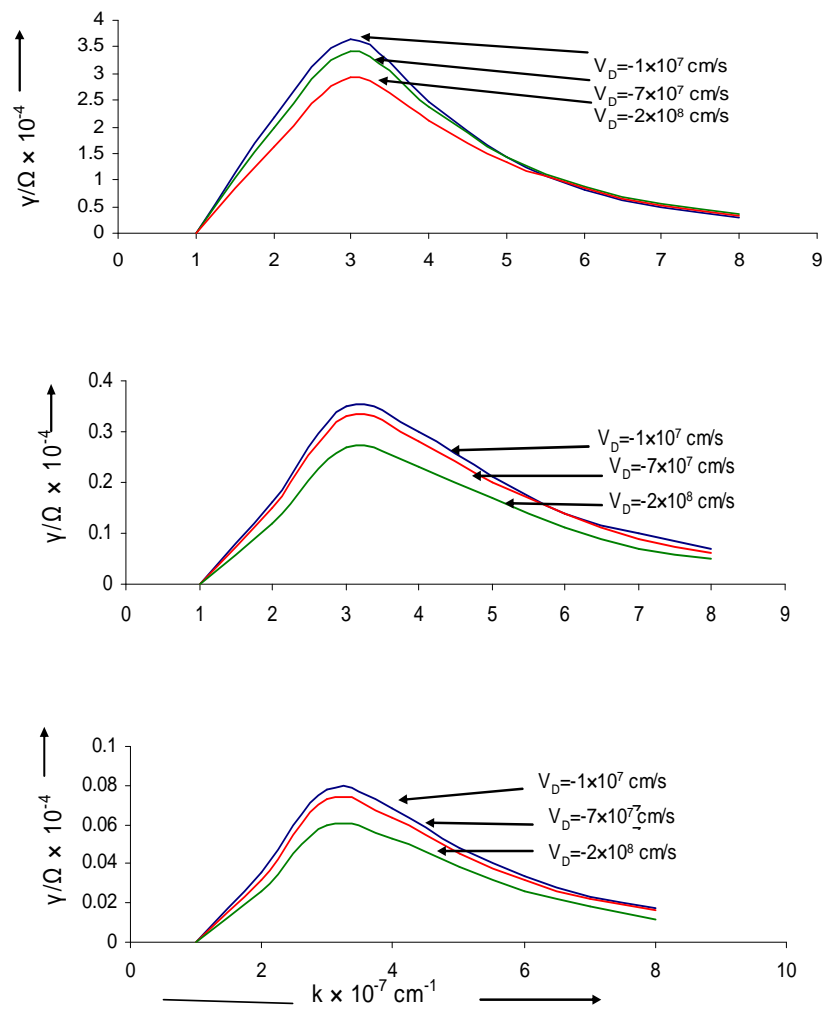

Fig. 1. Variation of Growth rate $(\gamma / \Omega)$ versus $\boldsymbol{k}$, for different values of $V_{\mathrm{D}}$ at fixed values of electron thermal anisotropy, $A_{\mathrm{e}}=3 \times 10^{-1}$ and ion thermal anisotropy, $A_{\mathrm{i}}=10$ for hot plasma at $J=2,1,0$, respectively.

presentation. In auroral acceleration region the EMIC wave instability is determined as predicted by above figures.

Figure 1 shows the variation of growth rate versus wave vector $\boldsymbol{k}$ at different distribution indices $J=0,1,2$ respectively and different ion beam velocity. It is assumed that the ion beam is directed from the ionosphere towards the magnetotail and therefore negative. It is observed that the effect of increasing the ion beam velocity is to reduce the growth rate that may be due to the transfer of wave energy to the beam. The steepness of loss-cone distribution increases the growth rate as reported earlier (Ahirwar et al., 2007). The increase in the loss-cone index shifts the peak of growth rate towards the lower side of the wave number $k$ and the peak value of the growth rate also increases. Thus, the mirror-like structure of the magnetosphere with a steep loss-cone distribution may be unstable for the EMIC wave emission.

Up going, counter streaming and down going field-aligned electron beams are also often associated with up flowing ions with canonical pitch angle distribution over the auroral region (Lund et al., 2000, 2001; Yoshioka et al., 2000). The electromagnetic ion-cyclotron wave generates a field-aligned electron (Temerin and Roth, 1986), but they accelerate electrons downward. In the present investigation it is noted that ion beam are also generated by EMIC wave opposite to the direction of the propagation of the EMIC wave.
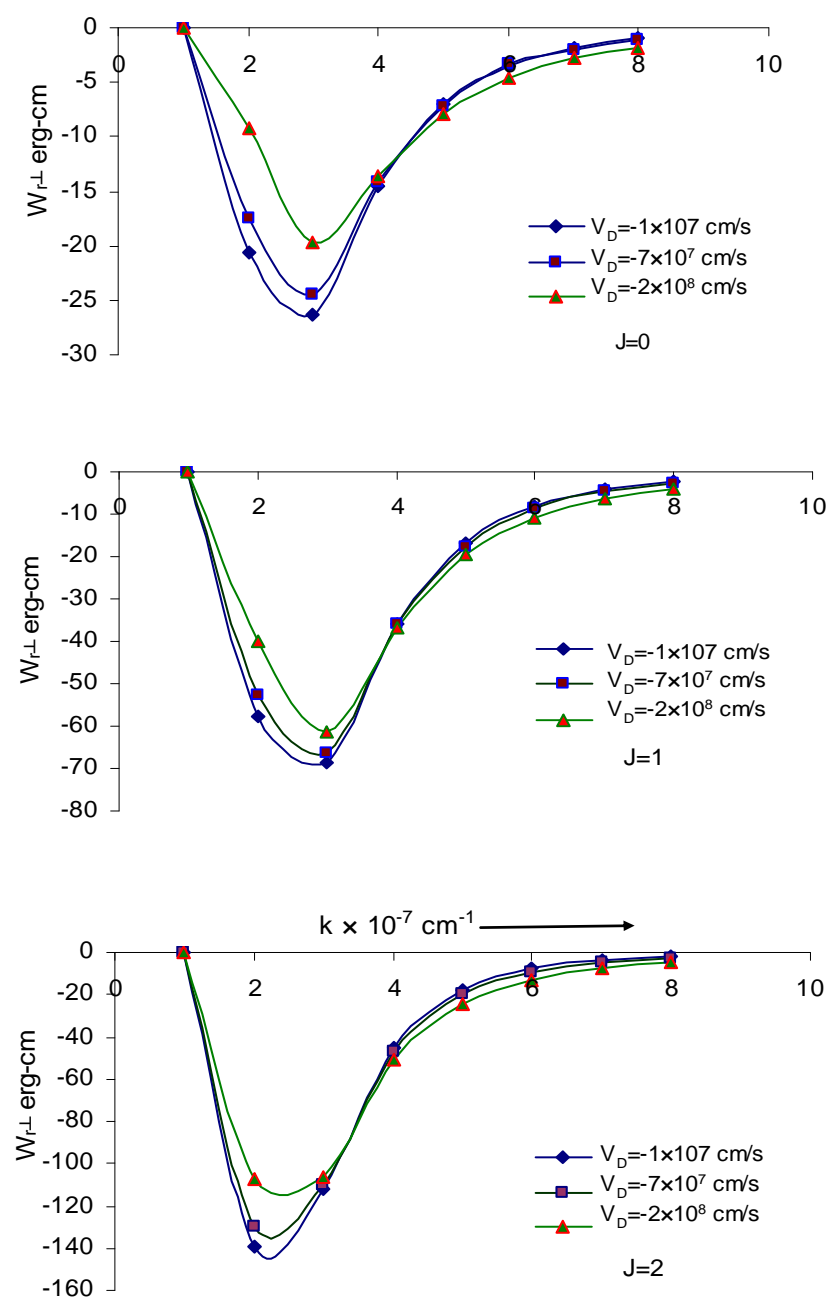

Fig. 2. Variation of perpendicular resonant energy $\left(W_{r \perp}\right)$ versus $\boldsymbol{k}$ at fixed values of electron thermal anisotropy, $A_{\mathrm{e}}=3 \times 10^{-1}$ and ion thermal anisotropy, $A_{\mathrm{i}}=10$ for hot plasma at $J=0,1,2$ for different values of $V_{\mathrm{D}}$.

Figure 2 depicts that the variations of perpendicular resonant energy $\left(W_{r \perp}\right)$ versus wave vector $\boldsymbol{k}$. This observation was found for different ion beam velocity at different distribution index $J$ for fixed ion and electron thermal anisotropy. When we increase the value of ion beam velocity the resonant energy in perpendicular direction decreases, the cause of it is that the ion beam extracts more energy from the wave and the perpendicular wave energy was consumed in EMIC wave excitation. The distribution index $J$ enhances the perpendicular resonant energy reduction as evident by the figure. The correlation of the EMIC ion conic with phenomena which are associated with ion thermal anisotropy play an significant role in transverse ion cooling in the aurora by the wave excitation. The particles heaving greater energy as compared to the wave transfer energy to the wave via cyclotron interaction and the wave growth is possible. The negative $W_{r \perp}$ indicates that the particle energy is transferred 

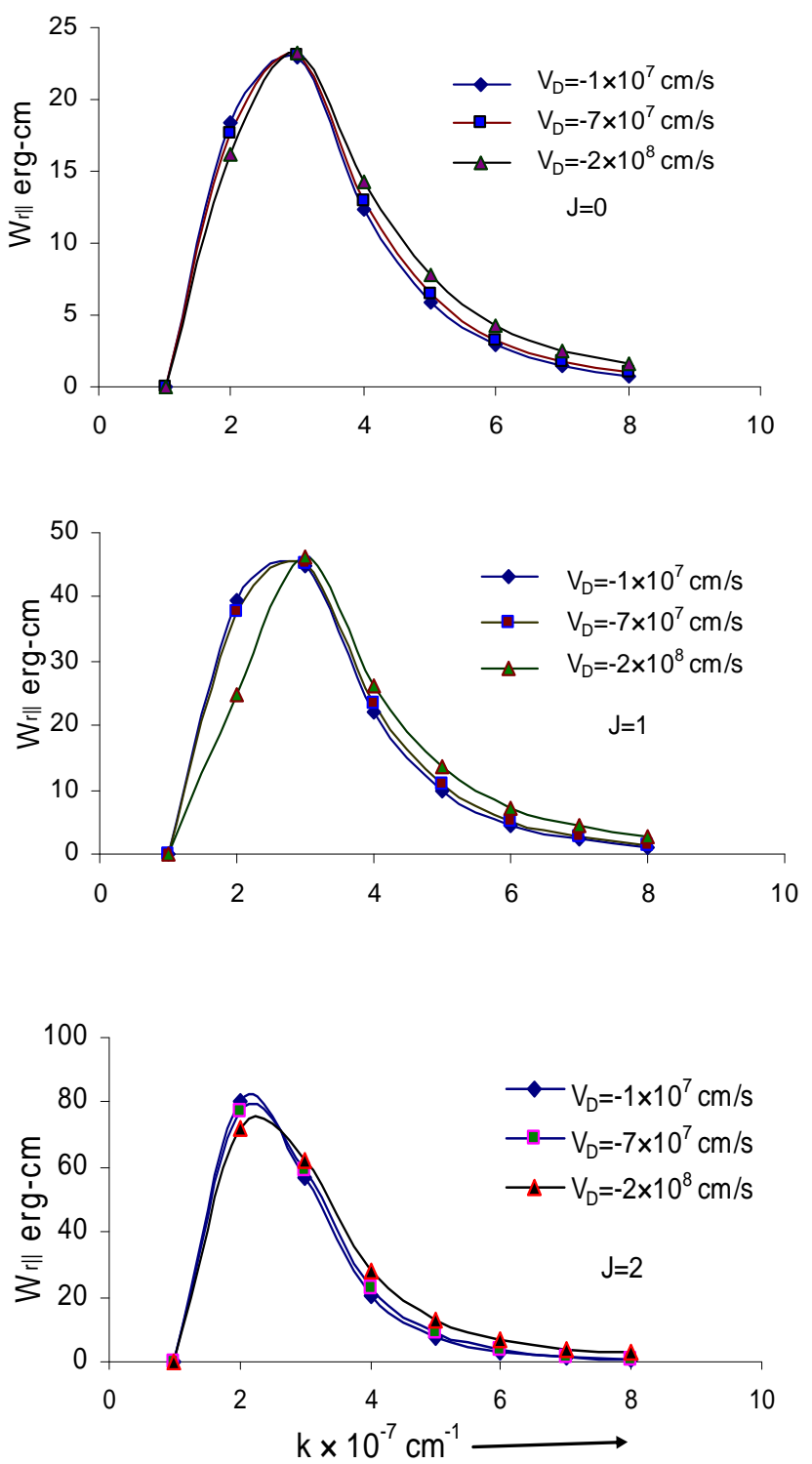

Fig. 3. Variation of parallel resonant energy $\left(W_{r \|}\right)$ versus $\boldsymbol{k}$ at fixed values of electron thermal anisotropy, $A_{\mathrm{e}}=3 \times 10^{-1}$ and ion thermal anisotropy, $A_{\mathrm{i}}=10$ for hot plasma at $J=0,1,2$ for different values of $V_{\mathrm{D}}$.

to the wave. Thus, wave emission occurs by extracting energy from the ion moving perpendicular to the magnetic field. Recently transversely accelerated ions and their association with EMIC waves have been reported by the analysis of FAST observations (Lund et al., 2000). The steep loss-cone decreases the energy of the transversely accelerated ions through the EMIC instability in the auroral acceleration region.

In Fig. 3 we showed the variation of parallel resonant energy versus wave vector $\boldsymbol{k}$. These figures exhibit parallel resonant energy of hot plasma at fixed electron thermal anisotropy $\left(A_{\mathrm{e}}\right)$ and ion thermal anisotropy $\left(A_{\mathrm{i}}\right)$ for distribu-

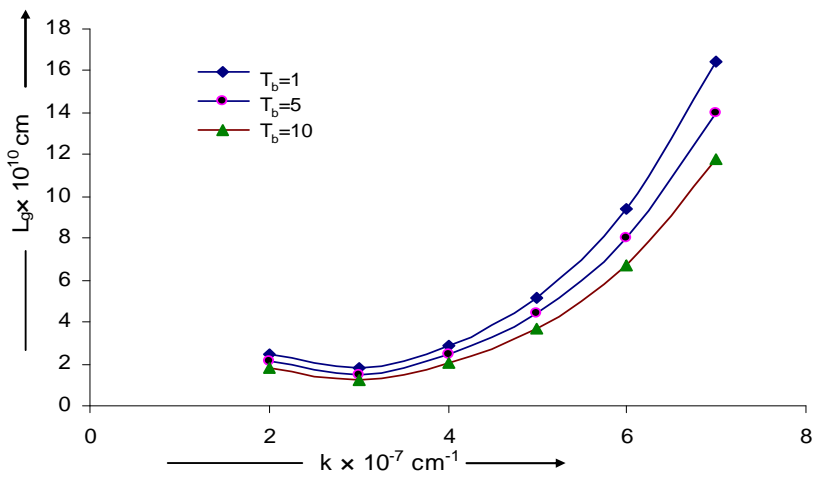

Fig. 4. Variation of growth length $\left(L_{\mathrm{g}}\right)$ versus wave vector $\boldsymbol{k}$, for different values of beam thermal anisotropies at fixed values of $A_{\mathrm{e}}=3 \times 10^{-1}$ and $A_{\mathrm{i}}=10$.

tion indices $J=0,1$ and 2 respectably. In this study we observed the effect of ion beam velocity on parallel resonant energy of hot plasma. The effect of increasing value of ion beam velocity is to decrease the parallel resonant energy through the EMIC wave. The effect of increasing value of distribution index $J$ is to increase the parallel resonant energy of the ions. This may be due to the mirroring effect of ions and interchange of energy between particle and wave.

Figure 4 indicates the variation of growth length $\left(L_{\mathrm{g}}\right)$ versus wave vector $\boldsymbol{k}$ at fixed values of electron thermal anisotropy, $A_{\mathrm{e}}=3 \times 10^{-1}$ and ion thermal anisotropy, $A_{\mathrm{i}}=$ 10 for hot plasma at $J=2$ for different values of beam thermal anisotropies. It is clear from figure that initially growth length decreases with increase in wave vector and after about $k=\sim 3 \times 10^{-7}$ growth length increases with the increase of wave vector and the increasing values of ion beam thermal anisotropy decrease the growth length of the waves. The growth length is in accordance to the growth rate, however the distance between the auroral acceleration region and the lower ionosphere is not sufficient for the wave to achieve its peak. The wave may not be completely excited/ dissipated by wave particle interaction mechanism and some other phenomena may be required.

Figure 5 shows the variation of Growth rate $(\gamma / \Omega)$, parallel resonant energy $\left(W_{r \|}\right)$, and perpendicular resonant energy $\left(W_{r \perp}\right)$ versus $\boldsymbol{k}$, for different values of beam thermal anisotropies $\left(T_{\mathrm{b}}\right)$ at fixed values of electron thermal anisotropy, $A_{\mathrm{e}}=3 \times 10^{-1}$ and ion thermal anisotropy, $A_{\mathrm{i}}=$ 10 for hot plasma at distribution index $J=2$. It is clear from figure that the growth rate and parallel energy increase with increasing the beam thermal anisotropy. Thus beam thermal anisotropy also contributes toward the growth of the EMIC wave. Whereas, there is a reduction in perpendicular energy increases with increasing beam thermal anisotropy, means that beam thermal anisotropy may be a source of energy for waves in earth's magnetosphere. 

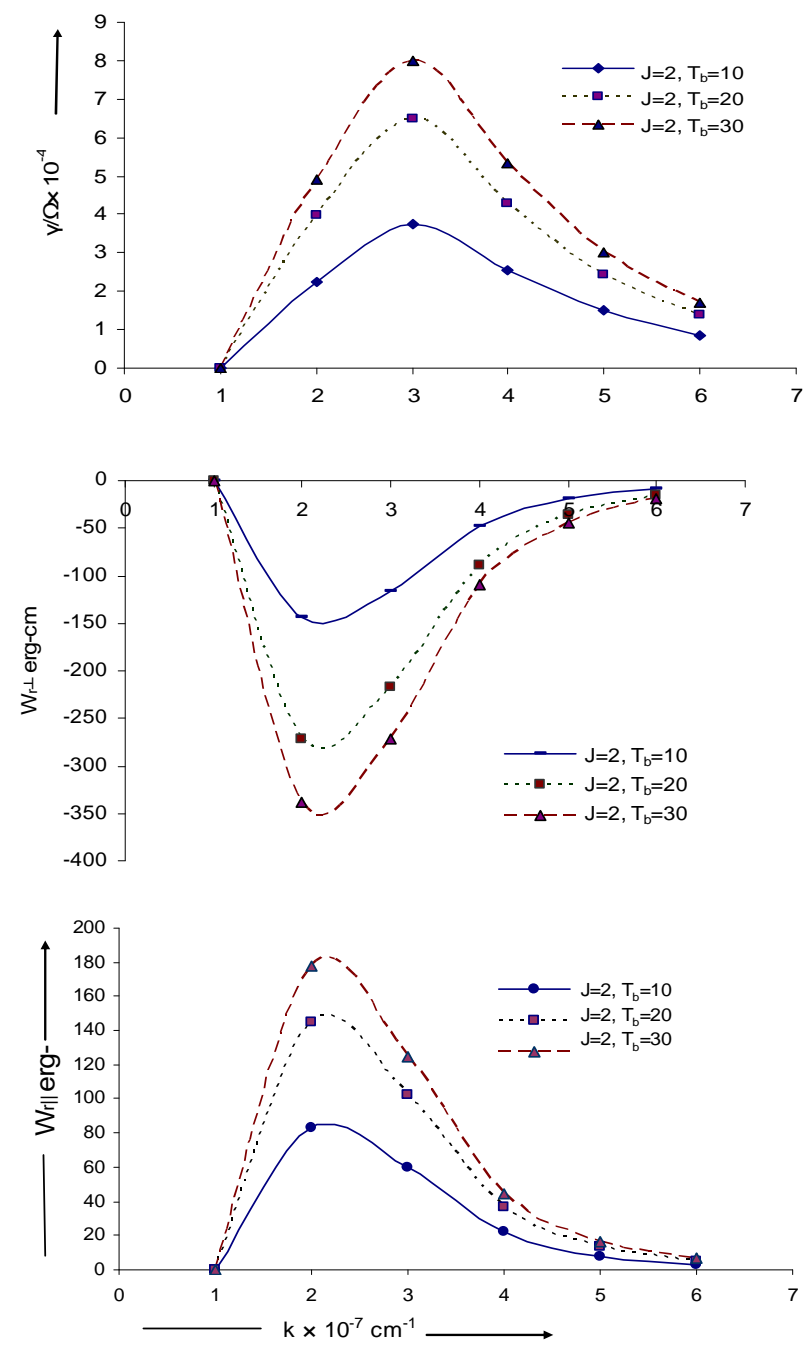

Fig. 5. Variation of Growth rate $(\gamma / \Omega)$, parallel resonant energy $\left(W_{r||}\right)$, perpendicular resonant energy $\left(W_{r \perp}\right)$ versus $\boldsymbol{k}$, for different values of beam thermal anisotropies at fixed values $A_{\mathrm{e}}=3 \times 10^{-1}$, $A_{\mathrm{i}}=10$ for hot plasma at $J=2$.

EMIC waves are important in magnetospheric dynamics since they are able to cause heating of the thermal plasma (Horne and Thorne, 1997), and pitch angle scattering and loss of both ring current ions and relativistic electrons. A quantitative assessment of the ability of EMIC waves to scatter relativistic electrons is complicated by the fact that, for a specified hot ion population, the most unstable excited wave frequencies tend to be lower (higher) in regions of higher (lower) density. EMIC waves also tend to have group velocities closely aligned with the magnetic field, which effectively prevents higher-frequency waves, excited in lowerdensity regions, from accessing high-density regions where the resonant electron energies are lower.

Figure 6 shows the variation of Growth rate, perpendicular and parallel energy versus wave vector $\boldsymbol{k}$ at fixed values of electron thermal anisotropy, $A_{\mathrm{e}}=3 \times 10^{-1}$ and ion ther-
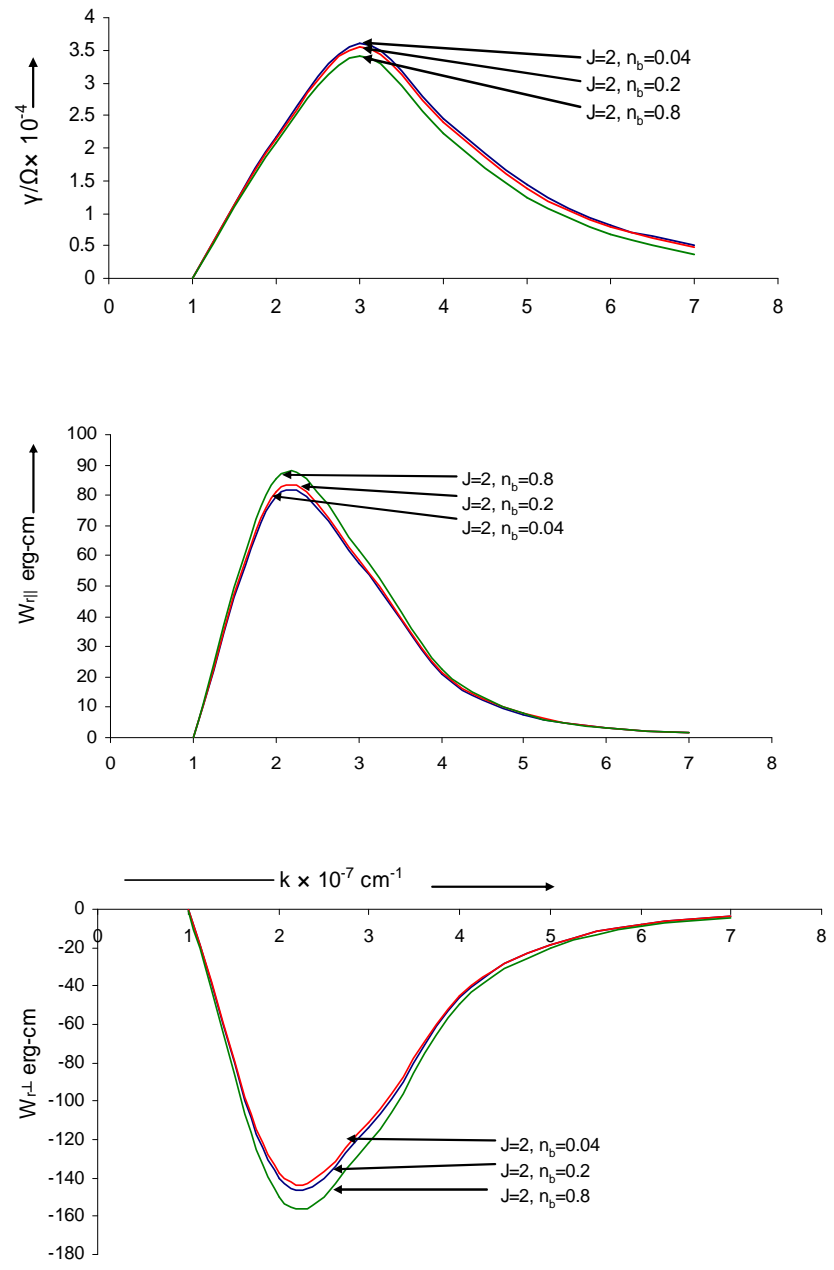

Fig. 6. Variation of Growth rate $(\gamma / \Omega)$, parallel resonant energy $\left(W_{r \|}\right)$, perpendicular resonant energy $\left(W_{r \perp}\right)$ versus wave vector $\boldsymbol{k}$, for different values of beam densities at fixed values of $A_{\mathrm{e}}=$ $3 \times 10^{-1}$ and $A_{\mathrm{i}}=10$ for hot plasma at $J=2$.

mal anisotropy, $A_{\mathrm{i}}=10$ for hot plasma at $J=2$ for different values of beam densities. It is clear that growth rate is decreased with increase in density of beam whereas parallel resonant energy and reduction in perpendicular resonant energies are increased with increase in beam density. Thus beam density also contributes similar to the beam velocity in the dynamic if EMIC wave. In this study we have considered only one ion species. However, in the auroral acceleration region, there are several ion species drifting relative to each other. The presence of various drifting ion affects the hot plasma dispersion relation (Cornwall and Schulz, 1971). The present basic model can be useful to explain some of the observations qualitatively and may be useful to explain perpendicular energisation in the extended solar corona. 


\section{Conclusion}

Present study indicates that hot anisotropic plasma may play a significant role in the description of auroral dynamic by EMIC instability. The role of ion beam velocity and beam anisotropy with loss-cone distribution function in hot anisotropic plasma should be taken into account of dealing the dynamics of EMIC wave in magnetospheric plasmas. This analysis indicates that beam anisotropy also play a source of energy for wave whereas, beam density, and beam velocities opposite to wave propagation control the dynamics of ion cyclotron waves significantly and may be the cause of dissipation of EMIC wave in the auroral acceleration region and growth length shows the length requires for wave excitation in hot plasma.

The perpendicular, parallel resonant energies, growth rate and growth length, explain the wave scenario in auroral acceleration region. It is applicable to explain EMIC wave phenomena in earth's magnetosphere. The effect of increasing ion beam velocity is to reduce the growth rate that may be due to a shifting of the resonance condition. This is an example demonstrating that EMIC waves serve as media for energy transfer from hot plasma to the cold plasma. The hot plasma provides the free energy for wave generation and the subsequent damping of these waves heats up the cold plasma (Fok, 2005). In view of the observations of EMIC wave around the auroral acceleration region our theoretical investigation indicate that the EMIC wave emission and the related phenomena can be suitably described considering the general loss-cone distribution function in the anisotropic hot plasma, which may be the cause of ring current destabilization and pitch angle scattering. The EMIC waves resulting in the magnetosphere are not only a passive element in the coupled ring current ionospheric system but also may influence the electrodynamics of coupling.

Acknowledgements. Financial assistance of UGC (to Soniya Patel), DST (to P. Varma) and ISRO (to M.S.T.) is thankfully acknowledged.

Guest Editor M. Gedalin thanks O. Onishchenko and two other anonymous referees for their help in evaluating this paper.

\section{References}

Ahirwar, G., Varma, P., and Tiwari, M. S.: Study of electromagnetic ion-cyclotron wave with general loss-cone distribution function, Ind. J. Phys., 80(12), 1179-1187, 2006a.

Ahirwar, G., Varma, P., and Tiwari, M. S.: Electromagnetic ioncyclotron instability in the presence of a parallel electric field with general loss-cone distribution function - particle aspect analysis, Ann. Geophys., 24, 1919-1930, doi:10.5194/angeo-241919-2006, 2006b.

Ahirwar, G., Varma, P., and Tiwari, M. S.: Beam effect on electromagnetic ion-cyclotron waves with general loss - cone distribution function in an anisotropic plasma-particle aspect analysis,
Ann. Geophys., 25, 557-568, doi:10.5194/angeo-25-557-2007, 2007.

Anderson, B. J., Erlandson, R. E., and Zanetti, L. J.: A statistical study of Pc 1-2 magnetic pulsations in the equatorial magnetosphere, 1, Equatorial occurrence distributions, J. Geophys. Res., 97, 3075-3088, 1992a.

Anderson, B. J., Erlandson, R. E., and Zanetti, L. J.: A statistical study of Pc 1-2 magnetic pulsations in the equatorial magnetosphere, 2, Wave properties, J. Geophys. Res., 97, 3089-3101, $1992 b$.

Braysy, T., Mursula, K., and Marklund, G.: Ion cyclotron waves during a great magnetic storm observed by Freja double-probe electric field instrument, J. Geophys. Res., 103, 4145-4155, 1998.

Cattell, C. A., Mozer, F. S., Roth, I., Anderson, R. R., Elphic, R. C., Lennartsson, W., and Ungstrup, E.: ISEE 1 observations of electrostatic ion cyclotron waves in association with ion beams on auroral field lines from $\sim 2.5$ to 4.5 Re, J. Geophys. Res., 96, 11421-11439, 1991.

Collin, H. L., Shelley, E. G., Ghielmetti, A. G., and Sherp, R. D.: Observations of transverse and parallel acceleration of terrestrial ions at high altitudes in ion acceleration in the magnetosphere and ionosphere, Tom Chang, American Geophysical Union, Washington, 67, 1986.

Collin, H. L., Peterson, W. K., Lennartsson, O. W., and Drake, J. F.: The seasonal variation of Auroral Ion Beams, Geophys. Res. Lett., 25(21), 4071-4074, 1998.

Cornwall, J. M. and Schulz, M.: Electromagnetic ion-cyclotron instabilities in multicomponent magnetospheric plasma, J. Geophys. Res., 76, 7791-7796, 1971.

Daughton, W. and Gary, S. P.: Electromagnetic proton/proton instabilities in the solar wind, J. Geophys. Res., 103(A9), 2061320620, 1998.

Duan, S. P., Li, Z, Y., and Liu, Z. X.: Kinetic Alfven waves driven by the density inhomogeneity in the presence of loss-cone distribution function-particle aspect analysis, Planet. Space Sci., 53, 1167-1173, 2005.

Engebretson, M. J., Kelling, A., Fornacon, K. H., Cattell, C. A., Johnson, J. R., Posch, J. L., Quick, S. R., Glassmeier, K. H., Parks, G. K., and Reme, H.: Cluster observations of Pc 1-2 waves and associated ion distributions during the October and November 2003 magneti storns, Planet. Space Sci., 55, 829-848, 2007.

Ergun, R. E., Carlson, C. W., McFadden, J. P., Mozer, F. S., Delory, G. T., Peria, W., Chaston, C. C., Temerin, M., Roth, I., Muschietti, L., Elphic, R., Strangeway, R., Pfaff, R., Cattell, C. A., Klumper, D., Shelley, E., Peterson, W., Moebius, E., and Kistler, L.: FAST satellite observations of large amplitude solitary structure, Geophys. Res. Lett., 25, 2041-2044, 1998.

Erlandson, R. E. and Ukhorskiy, A. J.: Observations of Electromagnetic ion cyclotron waves during geomagnetic storms: Wave occurrence and pitch angle scattering, J. Geophys. Res., 106, 38833895, 2001.

Erlandson, R. E., Zanetti, L. J., Potemra, T. A., Block, L. P., and Holmgren, G.: Viking magnetic and electric field observations of Pc 1 waves at high latitudes, J. Geophys. Res., 95, 5941-5955, 1990.

Fok, M. C., Kozyra, J. U., Nagy, A. F., Rasmussen, C. E., and Khazanov, G. V.: Decay of equatorial ring current ions and associated 
aeronomical consequences, J. Geophys. Res., 98, 19381-19393, 1993.

Fok, M. C., Ebihara, Y., and Moore, T. E.: Inner magnetospheric plasma interaction and coupling with the ionosphere, Adv. Polar Upper Atmos. Res., 19, 106-134, 2005.

Gamayunov, K. V., Khazanov, G. V., Liemhn, M. W., Fok, M. C., and Ridley, A. J.: Self-consistent model of magnetospheric electric field, ring current, plasmasphere, and electromagnetic ion cyclotron waves, Initial results, J. Geophys. Res., 114, A03221, doi:10.1029/2008JA013597, 2009.

Gomberoff, L.: Electrostatic waves in the Earth's magnetotail and in Comets and Electromagnetic instabilities in the magnetosphere and the solar wind, IEEE Transactions on Plasma Sci., 20, 843866, 1992.

Gomberoff, L. and Cuperman, S.: On the kinetic instability of uniform plasma with generalized loss-cone distribution function, J. Plasma Phys., 25, 99-102, 1981.

Gomberoff, L. and Elgueta, R.: Resonant acceleration of alpha particles by ion cyclotron waves in the solar wind, J. Geophys. Res., 96, 9801-9804, 1991.

Horne, R. B. and Thorne, R. M.: Wave heating of $\mathrm{He}^{+}$by electromagnetic ion cyclotron waves in the magnetosphere: heating near the $\mathrm{H}^{+}-\mathrm{He}^{+}$bi-ion resonance frequency, J. Geophys. Res. 102, 11457-11471, 1997.

Jardanova, V. K., Farrugia, C. J., Thorne, R. M., Khazanov, G. V., Reeves, G. D., and Thomsen, M. F.: Modeling ring current proton precipitation by electromagnetic ion cyclotron waves during the may 14-16, 1997, storm, J. Geophys. Res., 106, 7-22, 2001.

Kennel, C. F. and Petschek, H. E.: Limit on stably trapped particle fluxes, J. Geophys. Res., 71, 1-28, 1966.

Khazanov, G. V., Gamayunov, K. V., and Jardanova, V. K.: Selfconsistent model of magnetospheric ring current and electromagnetic ion cyclotron waves. The 2-7 may 1998 storm, J. Geophys. Res., 108(A12), 1419-1437, 2003.

Kozyra, J. U., Shelley, E. G., Confort, R. H., Brace, L. H., Cravens, T. E., and Nagy, A. F.: The role of ring current $\mathrm{O}^{+}$in the formation of stable auroral red arc, J. Geophys. Res., 92, 7487-7502, 1987.

Lund, E. J., Mobius, E., Ergun, R. E., and Carlson, C. W.: Massdependent effects in ion conic production: The role of parallel electric fields, Geophys. Res. Lett., 26, 3593-3596, 1999c.

Lund, E. J., Mobius, E., Klumper, D. M., Kistler, L. M., Popecki, M. A., Klecker, B., Ergun, R. E., McFadden, J. P., Carlson, C. W., and Strangeway, R. J.: Occurrence distribution of preferentially heating events in the aurora, Adv. Space Res., 23, 1721-1724, 1999a.

Lund, E. J., Mobius, E., Klumpar, D. M., Kistler, L. M., Popecki, M. A., Klecker, B., Ergun, R. E., McFadden, J. P., Carlson, C. W., and Strangeway, R. J.: Direct comparison of transverse ion acceleration mechanisms in the aurora region of solar minimum, J. Geophys. Res., 104, 22801-22805, 1999b.

Lund, E. J., Mobius, E., Carlso, C. W., Ergun, R. E., Kistler, L. M., Klecker, B., Klumpar, D. M., McFadden, J. P., Popecki, M. A., Strangerway, R. J., and Tung, Y. K.: Transverse ion acceleration mechanism in the aurora at solar minima: Occurrence distributions, J. Atmos. Solar-Terr. Phys., 62, 467-475, 2000.

Lund, E. J., Mobius, E., Lynch, K. A., Klumpar, D. M., Peterson, W. K., Ergun, R. E., and Carlson, C. W.: On the mass dependence of transverse ion acceleration by broad-band extremely low fre- quency waves, Phys. Chem. Earth, 26, 161-163, 2001.

Mauk, B. H.: Helium resonance and dispersion effects on geostationary Alfven/ion cyclotron waves, J. Geophys. Res., 87, 91079119, 1982.

Mishra, R. and Tiwari, M. S.: Effect of parallel electric field on electrostatic ion-cyclotron instability in anisotropic plasma in the presence of ion beam and general distribution function- particle aspect analysis, Planet. Space Sci., 54, 188-199, 2006.

Misra, K. D. and Tiwari, M. S.: Particle aspect analysis of electromagnetic ion-cyclotron instability, Can. J. Phys., 57, 1124-1130, 1979.

Mozer, F. S. and Hull, A.: The origin and geometry and upward parallel electric field in the auroral acceleration region, J. Geophys. Res., 106, 5763-5778, 2001.

Mozer, F. S., Carlson, C. W., Hudson, M. K., Torbert, R. B., Parady, B., and Yatteau, J.: Observations paired electrostatic shocks in the polar magnetosphere, Phys. Rev. Lett., 38, 292-295, 1977.

Newell, P. T., Meng, C. I., and Wing, S.: Relation to solar activity of intense aurorae in sunlight and darkness, Nature, 393, 342-344, 1998.

Patel, S., Varma, P., and Tiwari, M. S.: Comparative study between cold plasma and hot plasma with ion beam and loss-cone distribution function by particle aspect approach, Plasma Phys. Control. Fusion, 53, 035021-035036, 2011.

Sandanger, M., Soraas, F., Aarsnes, K., Oksavik, K., and Evans, D. S.: Loss of relativistic electrons: Evidence for pitch angle scattering by electromagnetic ion cyclotron waves excited by unstable ring current protons, J. Geophys. Res., 112, A12213, doi:10.1029/2006JA012138, 2007.

Sharp, R. D., Johnson, R. G., and Shelley, E. G.: Observation of an ionospheric mechanism producing energetic $(\mathrm{keV})$ ions primarily normal to the geomagnetic field direction, J. Geophys. Res., 82, 3324-3328, 1977.

Summers, D., Thorne, R. M., and Xiao, F.: Relativistic theory of wave-particle resonant diffusion with application to electron acceleration in the magnetosphere. J. Geophys. Res., 103(A9), 20487-20500, 1998.

Temerin, M. and Roth, I.: Ion heating by waves with frequencies below the ion gyrofrequency, Geophys. Res. Lett., 13, 1109-1115, 1986.

Thorne, R. M. and Horne, R. B.: Modulation of electromagnetic ion cyclotron instability due to interaction with ring current during magnetic storms, J. Geophys. Res., 102, 14155-14163, 1997.

Tiwari, B. V., Mishra, R., Varma, P., and Tiwari, M. S.: Sheardriven kinetic Alfven wave in the plasma sheet boundary layer, Earth Plan. Space, 60, 191-205, 2008.

Usanova, M. E., Mann, I. R., Rae, I. J., Kale, Z. C., Angelopoulos, V. Bonnell, J. W., Glassmeier, K. H., Auster, H. U., and Singer, H. J.: Multipoint observations of magnetospheric compressionreleted EMIC Pc1 waves by THEMIS and CARISMA, Geophys. Res. Lett., 35, L17S25, doi:10.1029/2008GL034458, 2008.

Yoshioka, R., Miyake, W., Mukai, T., and Ito, M.: Field-aligned electron beams observed simultaneously with upflowing ion beams in the auroral acceleration region, J. Geophys. Res., 105, 7679-7694, 2000. 